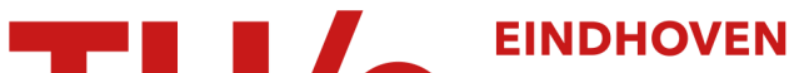 UNIVERSITY OF TECHNOLOGY
}

\section{Generic behaviour of grazing impact oscillators}

Citation for published version (APA):

Weger, de, J. G., Binks, D. J., Molenaar, J., \& Water, van de, W. (1996). Generic behaviour of grazing impact oscillators. Physical Review Letters, 76(21), 3951-3954. https://doi.org/10.1103/PhysRevLett.76.3951

DOI:

10.1103/PhysRevLett.76.3951

Document status and date:

Published: 01/01/1996

\section{Document Version:}

Publisher's PDF, also known as Version of Record (includes final page, issue and volume numbers)

\section{Please check the document version of this publication:}

- A submitted manuscript is the version of the article upon submission and before peer-review. There can be important differences between the submitted version and the official published version of record. People interested in the research are advised to contact the author for the final version of the publication, or visit the $\mathrm{DOI}$ to the publisher's website.

- The final author version and the galley proof are versions of the publication after peer review.

- The final published version features the final layout of the paper including the volume, issue and page numbers.

Link to publication

\section{General rights}

Copyright and moral rights for the publications made accessible in the public portal are retained by the authors and/or other copyright owners and it is a condition of accessing publications that users recognise and abide by the legal requirements associated with these rights.

- Users may download and print one copy of any publication from the public portal for the purpose of private study or research.

- You may not further distribute the material or use it for any profit-making activity or commercial gain

- You may freely distribute the URL identifying the publication in the public portal.

If the publication is distributed under the terms of Article $25 \mathrm{fa}$ of the Dutch Copyright Act, indicated by the "Taverne" license above, please follow below link for the End User Agreement:

www.tue.nl/taverne

\section{Take down policy}

If you believe that this document breaches copyright please contact us at:

openaccess@tue.nl

providing details and we will investigate your claim. 


\title{
Generic Behavior of Grazing Impact Oscillators
}

\author{
John de Weger, ${ }^{1}$ Doug Binks, ${ }^{1}$ Jaap Molenaar, ${ }^{2}$ and Willem van de Water ${ }^{1}$ \\ ${ }^{1}$ Physics Department, Eindhoven University of Technology, P.O. Box 513, 5600 MB, Eindhoven, The Netherlands \\ ${ }^{2}$ Mathematics Department, Eindhoven University of Technology, P.O. Box 513, 5600 MB, Eindhoven, The Netherlands
}

(Received 19 December 1995)

\begin{abstract}
We give experimental evidence for a new bifurcation structure that arises when smooth dynamical systems cross a boundary. Our experiment concerns a driven impacting leaf-spring oscillator with a very precise control of the driving amplitude. The results are in surprisingly good agreement with the predictions of a simple nonlinear mapping that is valid near grazing impact (i.e., impact with zero velocity). The agreement is surprising because a multitude of vibration modes of the spring is excited upon impact whereas the mapping is two dimensional. These findings point to the universality of the observed bifurcation structure. [S0031-9007(96)00276-1]
\end{abstract}

PACS numbers: 05.45.+b, 46.30.My, 46.30.Pa, 61.16.Ch

Impact oscillators have an oscillating mass that impacts with a fixed boundary when its amplitude is large enough. In between impacts the dynamics is smooth and often linear, but it derives a strong nonlinearity from the mere presence of the impact. These systems are known to exhibit a richness of bifurcation phenomena [1,2], but a strong guiding principle has, so far, been lacking. Impact oscillators are prototypical for nonlinear phenomena in engineering systems that are often designed with loose fittings. A timely application of impact oscillators is in atomic force microscopy [3].

A grazing impact is a boundary impact with zero velocity. In order to describe the events that occur when an orbit evolves to grazing impact and beyond, let us define $\rho$ as the bifurcation parameter. When the driving amplitude is increased from zero, impacts first occur at $\rho=0$. These impacts may be with a relatively large velocity and the transition that has taken place may be hysteretic; i.e., impacts may remain when $\rho$ is subsequently smoothly decreased and may vanish only at a negative value of $\rho$. When the impacts are about to disappear, the orbit is closest to grazing. In a recent paper Nordmark [4] has reduced the dynamics of impact oscillators to a simple nonlinear mapping that is valid for orbits close to grazing ones. A striking phenomenon in this mapping is the emergence of a square-root singularity.

An extensive bifurcation analysis of this mapping has recently been reported by Chin et al. [5]. Among the found characteristic phenomena are an infinite series of period addings for the overdamped oscillator and an infinite series of period-1 $\left(p_{1}\right)$ to period- $m\left(p_{m}\right), m=$ $3,4, \ldots$ transitions for the underdamped case. These phenomena are a direct consequence of the square-root singularity of the mapping and are independent of the details of the dynamics in between collisions. They are, therefore, predicted to be universal. In this Letter we present for the first time experimental evidence for one of these universal phenomena, namely, the infinite series of $p_{1}$ to $p_{m}$ transitions.

0031-9007/96/76(21)/3951(4)\$10.00
The experiment is sketched in Fig. 1. The oscillator consists of a $13 \mathrm{~cm}$ long brass leaf spring that is mounted on a large electromagnetic exciter which oscillates horizontally. The beam is weakly damped by strips of damping material that are glued to it. It is $\mathrm{U}$ shaped to suppress torsional modes but the excitation of higher-order bending modes is unavoidable. Collisions occur between a ceramic ball that is attached to the end of the beam and a hardened steel plate that is attached to the exciter. The motion of the beam is registered by reflecting a laser beam off the beam onto a position sensitive photodiode. The measurement and control of the excitation amplitude is a crucial aspect of the experiment. The excitation amplitude (typically $2 \mathrm{~mm}$ ) is measured using a laser interferometer and fringe counter and is digitally controlled with a long-term stability of $0.5 \mu \mathrm{m}[6]$.

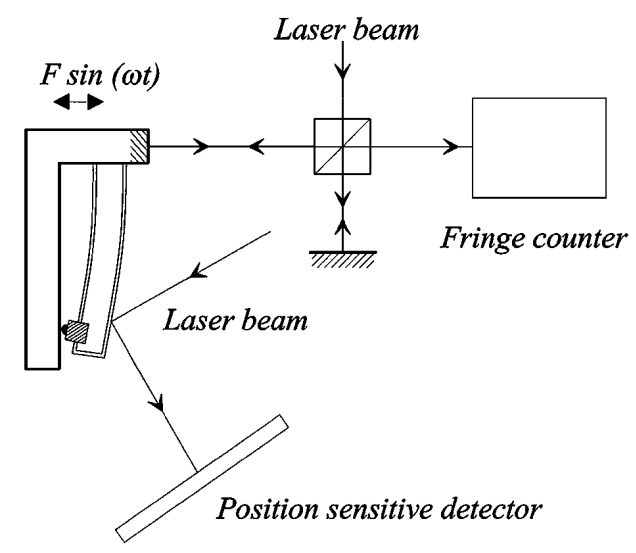

FIG. 1. A U-shaped leaf spring is brought into oscillation by horizontally oscillating its support. At a large enough forcing amplitude $F$, the attached mass impacts with a stop. Collisions take place between a hard ceramic ball and a hardened steel plate. The amplitude of the exciter is measured interferometrically. The deflection of the leaf spring is registered by reflecting a laser beam off the spring onto a position sensitive diode. 
The experiment is approximately described by the differential equation (overdots denote time derivatives)

$$
\ddot{\xi}+\mu \dot{\xi}+\Omega^{2} \xi=F \sin (\omega t),
$$

where $\Omega$ is the eigenfrequency of the free oscillator, $\mu$ represents the damping, and the excitation force has amplitude $F$ and frequency $\omega$. When the position $\xi(t)$ comes to the boundary at, say, $t_{0}$ the velocity reflects as $\dot{\xi}\left(t_{0^{+}}\right)=-\tau \dot{\xi}\left(t_{0^{-}}\right)$, where $\tau$ is the restitution coefficient. Equation (1) is approximate in that small oscillation amplitudes are assumed and, more importantly, higherorder modes of the beam are ignored. For near-grazing orbits in a broad class of second-order dynamical systems [one of which would be Eq. (1)] the following mapping can be derived [4] (see also [5]):

$$
\begin{array}{ll}
x_{n+1}=\alpha x_{n}+y_{n}+\rho & \text { for } x_{n} \leq 0 \\
y_{n+1}=-\gamma x_{n} & \\
x_{n+1}=-\sqrt{x_{n}}+y_{n}+\rho & \text { for } x_{n}>0, \\
y_{n+1}=-\gamma \tau^{2} x_{n} &
\end{array}
$$

where $x_{n}$ and $y_{n}$ are transformed coordinates of the $(\xi, \dot{\xi})$ space at stroboscopic times $t_{n}=n 2 \pi / \omega$, and where $\rho$ is the bifurcation parameter that measures the distance to the point of grazing impact. If no collision occurs between $t_{n}$ and $t_{n+1}$, the linear map Eq. (2) applies, whereas Eq. (3) describes the dynamics in the case that an impact will occur on $\left[t_{n}, t_{n+1}\right]$. For oscillators described by Eq. (1), the parameters of the mapping can be expressed explicitly in those of the differential equation, $\gamma=\exp (-\mu / f), \quad \alpha=2 \gamma^{1 / 2} \cos (2 \pi / f T)$ [5], and $\rho=\left(F / F_{g}-1\right)[1-\alpha+\gamma] / 2 \gamma(f T)^{2}(1+$ $\tau)^{2} \sin ^{2}(2 \pi / f T)$, where $f=\omega / 2 \pi$ is the excitation frequency, $T=2 \pi /\left[\Omega^{2}-(\mu / 2)^{2}\right]^{1 / 2}$ is the period of the free damped oscillations of the beam, and $F_{g}$ is the excitation amplitude at grazing impact. However, we emphasize again that Eqs. (2) and (3) apply to more general impacting oscillators than those described by Eq. (1). The presence of the square root in Eq. (3) is a key aspect of the mapping; it causes the Jacobian to be singular [7] at $x_{n}=0$, and it gives rise to the characteristic bordercollision bifurcations [8] that are reported in this Letter. The nature of the square root is simply the relation between elapsed time and traveled distance in systems with constant acceleration but the precise derivation of the mapping is highly nontrivial.

Our experiment corresponds to the underdamped case $\Omega^{2}>(\mu / 2)^{2}$ and analysis of the mapping predicts an infinite series of $p_{m}(m=3, \ldots, \infty)$, saddle-node bifurcations at $\rho=\rho_{m} \leq 0$ as the parameter $\alpha$ is varied from 0 to $2 \sqrt{\gamma}$ [9]. The stable period $m$ orbit has a single impact per period and is called a maximal periodic orbit [5]. At $\rho=0$ the unstable $m$ saddle collides with the $p_{1}$ orbit. Because the $p_{m}$ orbit is born away from the $p_{1}$ orbit, the transition $p_{1} \leftrightarrow p_{m}$ appears to be hysteretic: Whereas the transition $p_{1} \leftrightarrow p_{m}$ takes place at $\rho=0$, the transition $p_{m} \leftrightarrow p_{1}$ in a smooth downward scan of $\rho$ takes place at $\rho_{m} \leq 0$.
A measured series of transitions $p_{1} \leftrightarrow p_{m}$ for $m=$ 3 to $m=10$ is shown in Fig. 2. In our experiments the period-1 orbit is the trivial periodic motion of the noncolliding beam. The highest observed period $\left(p_{10}\right)$ is at the limit of our experimental resolution and stability. The shown series of bifurcations accurately follows the prediction of the mapping.

The $p_{1} \leftrightarrow p_{m}$ transitions occur in bands in the $(\alpha, \gamma)$ parameter plane of the mapping [5] that are crossed approximately transversely by scanning the excitation frequency in our experiment. At the upper boundary of the $m$ region, the $p_{m}$ saddle node is born exactly at $\rho=0$ and there is no hysteresis. The hysteresis increases towards the upper boundary of the lower-lying $m-1$ boundary. Figure 3 compares the predicted hysteresis $F_{m} / F_{g}-1$ with that found experimentally, where $F_{m}$ is the excitation amplitude at which the period- $m$ orbit vanishes in a smooth downward scan of $F$. All experimental resonances are found in place, but the measured hysteresis differs from the prediction. We believe that the discrepancy is due to higher-order vibrational modes of the beam.

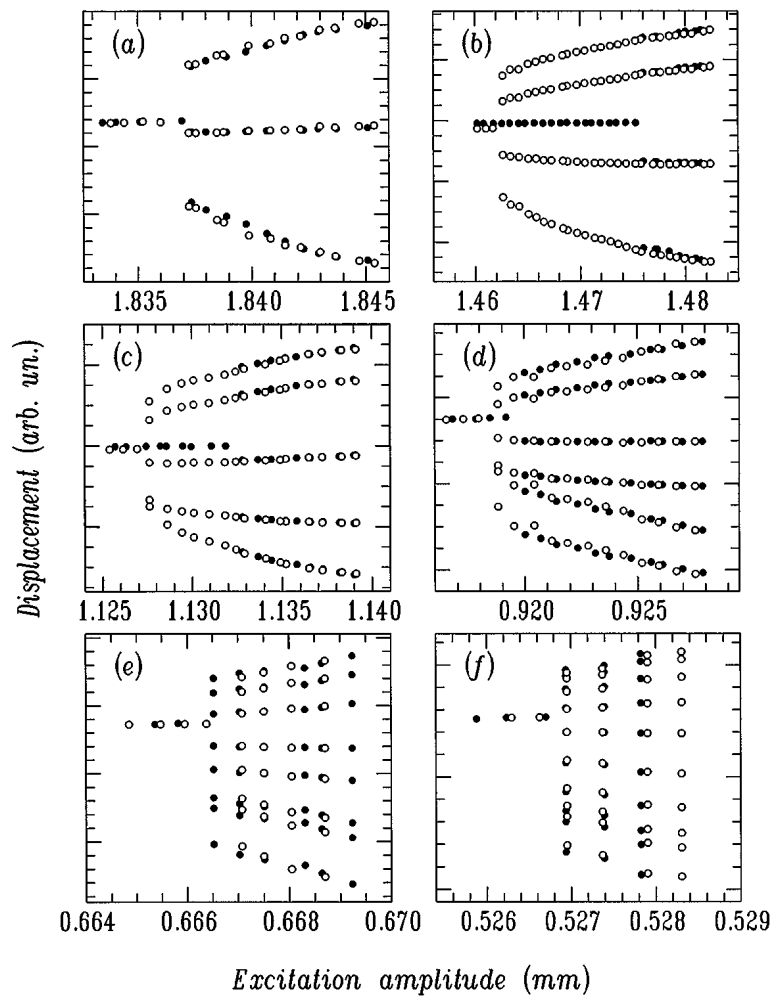

FIG. 2. Experimental bifurcation diagrams of $p_{1} \leftrightarrow p_{m}$ transitions with $m=3,4,5,6,8,10$ for (a)-(f), respectively. The displacement of the beam is measured at stroboscopic times $t_{n}=n 2 \pi / \omega$. The closed circles are for the upward scan of the excitation amplitude; the open circles are for the downward scan. The period of the free swinging beam is $T=$ $40.80 \pm 0.02 \mathrm{~ms}$. The excitation frequencies are $f=20.90$, $21.50,22.20,22.60,23.05$, and $23.40 \mathrm{~Hz}$, for (a)-(f), respectively. In all cases the observed periodicity agrees with the prediction from the Nordmark map [Eqs. (2) and (3)]. 


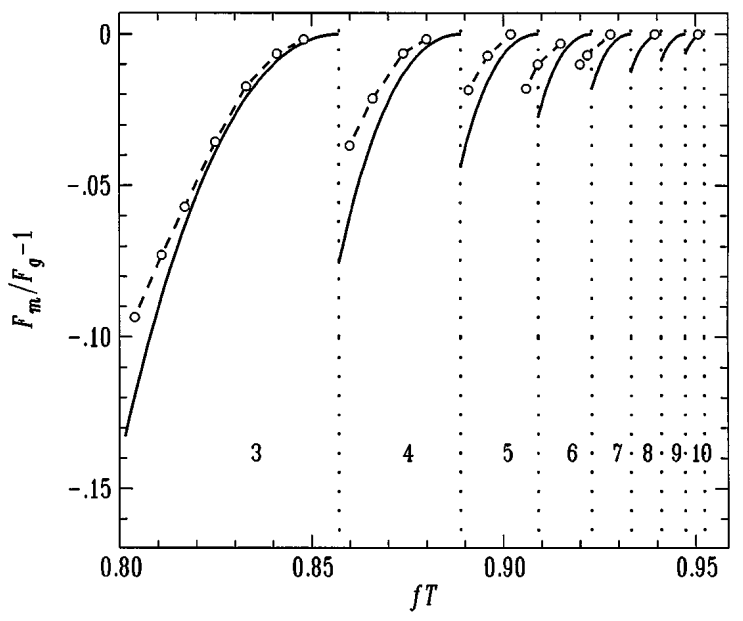

FIG. 3. Hysteresis of "maximal" $p_{m}$ orbits as a function of excitation frequency. Full lines: as computed from the Nordmark map Eqs. (2) and (3). Dotted lines: stability boundaries of the $p_{m}$ orbits as predicted by the Nordmark map; the corresponding periods $m$ are indicated. Open circles connected by dashed lines: experimental results. In a few cases $p_{m}$ orbits continue to exist in the lower-lying $p_{m-1}$ regions. This coexistence is actually predicted by the mapping Eqs. (2) and (3).

The excitation of higher-order modes upon impact is a characteristic feature of impacting in continuous systems. The energy in these modes is quickly dissipated, for example, by the radiation of sound. As a consequence, the collision is highly inelastic. This is accounted for by a nonunity restitution coefficient $\tau$. The presence of a multitude of higher-order modes on impact is demonstrated in Fig. 4, which shows the measured deflection as a function of time for a period-4 orbit. It therefore appears that the dynamics in our experiment is much more complex than described by Eq. (1) and the very favorable agreement with the mapping becomes a surprise. We will show that it is, perhaps paradoxically, precisely one of the nonidealities of the experiment that makes the mapping more appropriate.

It is important to realize that the mapping is derived for orbits that have a collision that is near grazing and the predicted bifurcations occur when the orbit near a particular grazing collision point evolves from near grazing to nonimpacting and back again when a parameter is smoothly varied. The proximity of an orbit to one containing a grazing collision is, therefore, the key parameter that determines the applicability of the mapping. Obviously, a $p_{m}$ orbit that results from a $p_{1} \rightarrow$ $p_{m}$ bifurcation with a large hysteresis will at $\rho=0$ be far away from a grazing collision; the distance increases as the hysteresis becomes larger.

The magnitude of the hysteresis $\left|\rho_{m}\right|$ decreases with increasing energy loss per collision. Therefore, for impacting systems with a large energy loss at each impact, the mapping [Eqs. (2) and (3)] is applicable in larger areas of parameter space than for impacting systems with smaller energy loss. In our experiment the restitution coefficient is

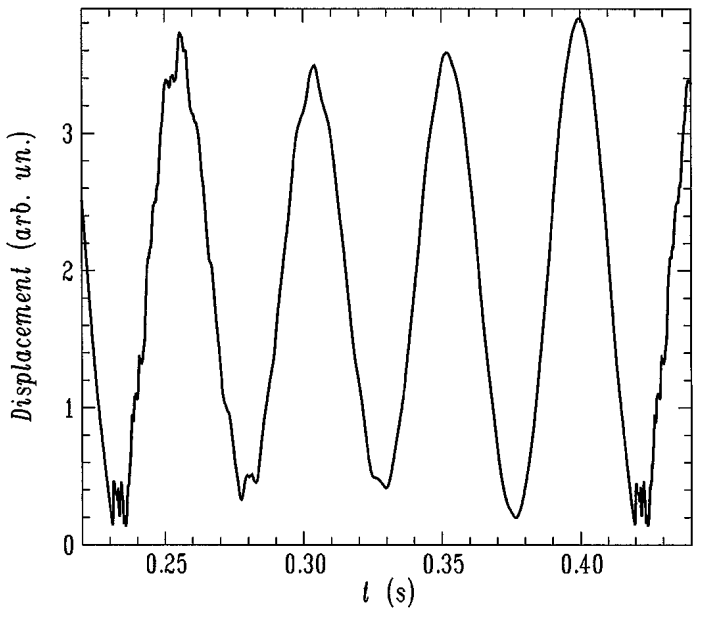

FIG. 4. Time trace of a period-4 orbit at $\rho=F / F_{g}-1=$ 0.013. At impact a multitude of high-order modes is excited in the beam.

estimated to be $\tau \approx 0.1$ and the $p_{1} \rightarrow p_{m}$ bifurcations could be found in a fairly large region of the predicted stability band in the $(\alpha, \gamma)$ plane. Outside this region, i.e., towards the upper stability boundary of the $p_{m-1}$ orbit, other transitions than the predicted $p_{1} \rightarrow p_{m}$ bifurcation occur; these may even be to chaotic states. In these cases the $p_{m}$ stable periodic orbit is found only at some negative value of $\rho$ and the $p_{m} \rightarrow p_{1}$ bifurcation is encountered when $\rho$ is decreased further. These other transitions are caused by secondary bifurcations on $\rho_{m}<\rho<0$ that are due to second impacts. In the mapping these secondary bifurcations always occur at $\rho>0$. In Eq. (1) secondary bifurcations may occur at values $\rho<0$ that become smaller if collisions become more elastic.

The breakdown of the mapping near $\rho=0$ is illustrated in Fig. 5, which shows an experimental bifurcation diagram at parameter values where the mapping predicts the $p_{3}$ orbit to be stable. At $\rho=0$ the trivial noncolliding $p_{1}$ orbit gives way to a period-6 orbit. The stable periodic $p_{3}$ orbit is found only at the very end of the downward scan of the driving amplitude. Incidentally, Eq. (1) predicts for this case a chaotic state at $\rho=-0.0707$ that is not connected to the state that is born at $\rho=0$. Clearly, the experimental result is also strongly influenced by the excitation of higher-order modes. The prediction of the mapping applies only when the orbit is closest to grazing.

The observed bifurcation phenomena are a consequence of the square-root singularity of the mapping. This singularity may be smoothed by the excitation of higherorder modes that introduce a time delay at impact. A crude model results if the impacts of the system described by Eq. (1) are imaged to occur with a soft wall such that at impact the spring constant changes by a factor $K \gg 1$ [10]. We have studied numerically the influence of a finite value of $K$ on the observed bifurcation phenomena [11]. A large value of $K$ affects the bifurcations close 


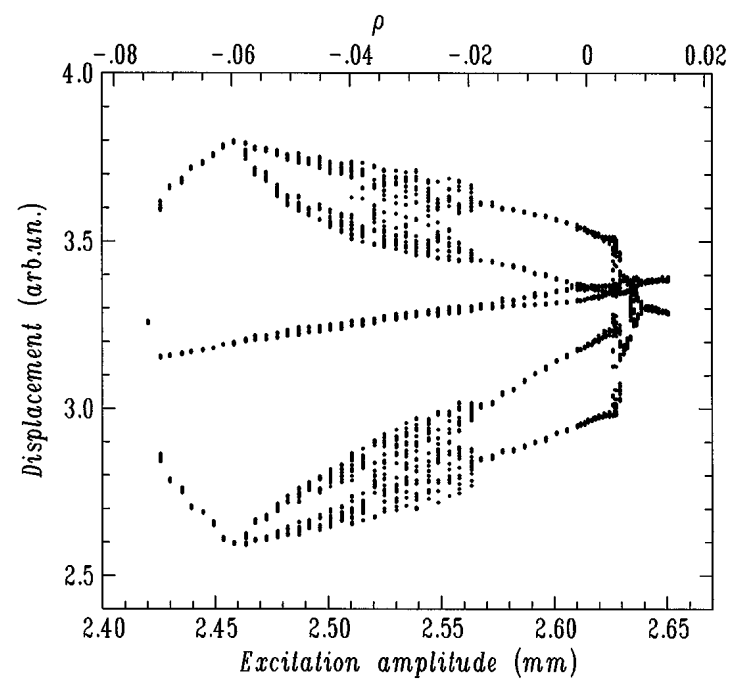

FIG. 5. Experimental bifurcation diagram measured in the region where the mapping predicts the $p_{1} \leftrightarrow p_{3}$ transition. For positive values of $\rho$ finer excitation amplitude steps are taken. At each amplitude setting the system is allowed to equilibrate for $\approx 10^{4}$ cycles after which 40 stroboscopic points are drawn. The excitation frequency is $f=19.65 \mathrm{~Hz}$, the measured period of the freely swinging beam is $T=41.2 \pm 0.1 \mathrm{~ms}$, and the damping constant $\mu=3.56 \pm 0.1 \mathrm{~s}^{-1}$.

to $\rho=0$ in a characteristic manner. The influence of the wall softness extends to larger values of $\rho$ as $K$ decreases. As we have not observed these secondary bifurcations in our experiment, we conclude that the excitation of higher order modes does affect the energy loss of a collision, but does not effectively smooth the singularity. The regularization of the singularity is an interesting problem and will be discussed in a forthcoming paper.

In conclusion, we have given experimental evidence for a new class of universal bifurcations that arises when smooth dynamical systems cross a boundary. These bifurcations are predicted by a mapping that reduces the dynamics to its essentials: namely, the occurrence of a singularity. Surprisingly, our evidence has been aided by the increased damping in the experiment that is a consequence of the excitation of higher-order modes. We expect that these phenomena should be observed in a wide class of experimental boundary crossing systems. The energy loss and time delay at impact are two parameters to effectively represent higher-order modes in a singlemode description. Obviously, when impacts are hard and these modes become an essential part of the dynamics, the analysis presented here no longer applies.
We thank Jan Niessen for technical assistance. We also would like to thank Dick van Campen and Bram de Kraker for having made possible initial experiments. We gratefully acknowledge financial support by the "Nederlandse Organisatie voor Wetenschappelijk Onderzoek (NWO)."

[1] S. W. Shaw and P. Holmes, Phys. Rev. Lett. 51, 623 (1983); J. Sound Vib. 90, 129 (1983).

[2] S. W. Shaw, J. Sound Vib. 99, 199 (1985).

[3] Q. Zhong, D. Inniss, K. Kjoller, and V.B. Ellings, Surf. Sci. 290, L688 (1993); J. P. Spatz, S. Sheiko, M. Möller, R. G. Winkler, P. Reineker, and O. Marti, Nanotechnology 6, 40 (1995). A better understanding of impact oscillators may lead to a significant improvement of the sensitivity of atomic force microscopy.

[4] A. B. Nordmark, J. Sound Vib. 145, 279 (1991).

[5] W. Chin, E. Ott, H. E. Nusse, and C. Grebogi, Phys. Rev. E 50, 4427 (1994).

[6] The amplitude is controlled such that in amplitude scans the measured amplitude changes strictly monotonically. This is important for a correct assessment of the apparent hysteresis. Because the viscoelastic properties of the damping material are temperature dependent, the temperature needed to be kept constant to within $0.02{ }^{\circ} \mathrm{C}$.

[7] Singularities in this system in a more general context are discussed in G. S. Whiston, J. Sound Vib. 152, 427 (1992).

[8] H. E. Nusse and J. A. Yorke, Physica (Amsterdam) 57D, 39, 1992; H. E. Nusse, E. Ott, and J. A. Yorke, Phys. Rev. E 49, 1073 (1994).

[9] The $p_{1} \rightarrow p_{2}$ transition occurs at $-2 \sqrt{\gamma}<\alpha<0$. In addition, the transition $p_{1} \rightarrow p_{1}$, that is the bifurcation to a (grazing) impact orbit with period 1, is predicted by the mapping Eq. (3), but with a plus sign in front of the $\sqrt{x}$ term. Both predicted $p_{1} \rightarrow p_{1}$ and $p_{1} \rightarrow p_{2}$ transitions agree with experimental results. These phenomena will be discussed in detail in a forthcoming paper.

[10] A very similar model, but not for grazing collisions, was studied in [2].

[11] An efficient numerical scheme for solving Eq. (1) in the presence of grazing impacts uses the analytical solutions for the motion between impacts. The (exact) positions $\xi(t)$ are computed in a small number of discrete points $t_{1}, \ldots, t_{k}$ in each drive period. It is crucial not to miss boundary crossings of $\xi(t)$. These crossings are detected both directly by checking $\xi\left(t_{1}\right), \ldots, \xi\left(t_{k}\right)$ and by computing the position $\xi(t)$ at the turning points of the velocity $\dot{\xi}$. 